\title{
Akinetic-rigid syndrome: An unusual presentation of hypoglycemic encephalopathy
}

\author{
Akhila Kumar Panda, Kushwaha Suman, Aldrin Anthony Dung Dung
}

\begin{abstract}
Introduction: Hypoglycemia is an emergency. If untreated, it can result persistent neuronal injury of the central nervous system. The neurologic manifestations of hypoglycemia ranges from headache, seizure, different neuropsychiatric symptoms to stupurous, coma and stroke like symptoms. Akinetic-rigid syndrome, a severe form of extra pyramidal symptom is rarely described with hypoglycemic brain damage. The magnetic resonance imaging (MRI) findings are variable and reversible which ranges from normal study to diffusion restriction and white matter hyper intensities involving both cortical and sub cortical white matter as well as grey matter. Case Report: This case illustrates a 32-year-old male with history of intermittent hypoglycemia of unknown cause for three years duration presenting with prolonged akinetic mutism and extra pyramidal symptoms. His brain MRI revealed bilateral symmetrical white matter hyper intensities involving corona radiata, internal capsule and
\end{abstract}

Akhila Kumar Panda1 ${ }^{1}$ Kushwaha Suman², Aldrin Anthony Dung Dung ${ }^{3}$

Affiliations: ${ }^{1} \mathrm{MD}$, D.M, Neurology, Senior Resident, Department of Neurology, Institute of Human Behaviour and Allied Sciences (IHBAS), Delhi, India; ${ }^{2 M D}$, D.M. Neurology, Associate Professor, Department of Neurology, Institute of Human Behaviour and Allied Sciences (IHBAS), Delhi, India; ${ }^{3} \mathrm{MD}$, DNB (Neurology), Assistant professor, Department of neurology, Institute of Human Behaviour and Allied Science (IHBAS), Delhi.

Corresponding Author: Akhila Kumar Panda, Type IV, Quarter No-12, IHBAS Hospital, Dilshad Garden, Delhi, India 110095; Ph: 09868396908, 09582524229; Email: drakhilapanda@gmail.com

Received: 23 November 2012

Accepted: 12 January 2013

Published: 01 August 2013 middle cerebellar peduncles with $18 F-$ fluorodeoxyglucose positron emission tomography with computed tomography (FDG PET CT) scan of brain showed hypometabolism involving bilateral parietal lobes, basal ganglia, thalamus and cerebellum, which reversed with correction of blood sugar within next four weeks. Conclusion: Hypoglycemic encephalopathy (HE) may develop reversible extra pyramidal syndromes. Clinico-neuro imaging correlation is not always possible. Besides routine evaluation for hypoglycemia, neuroimaging such as MRI of brain and 18FFDG PET CT scan may contribute to recognize the disease process and its outcome.

Keywords: Encephalopathy, Hypoglycemia, Cerebellar peduncle, Mutism, Akinetic-rigid syndrome

$$
* * * * * * * * *
$$

Panda AK, Suman K, Dung AAD. Akinetic-rigid syndrome: An unusual presentation of hypoglycemic encephalopathy. International Journal of Case Reports and Images 2013;4(8):427-430.

$* * * * * * * * *$

doi:10.5348/ijcri-2013-08-348-CR-7

\section{INTRODUCTION}

Cerebral hypoglycemia causes reversible metabolic brain insults. About $10 \%$ of type 2 and $4 \%$ in type 1 diabetes mellitus (DM) patients experienced severe hypoglycemia during natural course of the disease $[1,2]$. The common neurological manifestations of hypoglycemia are confusion, 'bizarre' behavior, stupurous, coma, seizure, cognitive impairment, 
headache, stroke like symptoms and generalized weakness [3, 4]. Extra pyramidal syndromes such as akinetic-rigid syndrome and mutisim are the less appreciated cause of hypoglycemic brain damage.

\section{CASE REPORT}

A 30-year-old male laborer presented with recurrent episodes of unconsciousness for last three years. These episodes were preceded by perioral and hand numbness followed by diaphoresis, cool extremities. These episodes were persisting for several hours. Such events usually occur in the working field in the late period of daytime and at night. Several times in the early morning he was found unconscious along with severe perspiration. Initially, the frequency was two to three episodes per month which was gradually increased to one every week for last three months. Fifteen days prior to admission, he developed prolonged state of delirium. He was initially misdiagnosed as generalized epilepsy. General physical examinations showed patient was mute with spontaneous reflex eye movements, none communicating to either verbal or gestural commands with normal vital parameters. Cardiovascular, respiratory and gastrointestinal examinations were unremarkable. Nervous system examinations revealed, the patient was mute with spontaneous non purposeful eye blinking and eye movement with normal oculocephalic reflex. Overall cranial nerves examinations including fundus were non contributory. Motor system examinations showed severe generalized lead pipe rigidity involving appendicular as well as axial group of muscles with paucity of spontaneous limb movements, brisk deep tendon reflexes and flexor plantar responses. Detail sensory and cerebellar examinations were not possible. There was no evidence of autonomic instability.

\section{LABORATORY INVESTIGATIONS}

The patient had persistent hypoglycemia of random capillary blood glucose ranging from $35^{-60} \mathrm{mg} / \mathrm{dL}$ despite continuous intravenous $5 \%$ dextrose solution. Other blood parameters including liver function, kidney function, thyroid profile, complete blood count were unremarkable. His serum for human immune deficiency virus (HIV), hepatitis B and C and syphilis were negative. Cerebrospinal fluid (CSF) study showed acellular background with normal protein and sugar values. CSF polymerase chain reactions (PCR) for herpes simplex virus and antibody analysis for Japanese encephalitis and West Nile virus were negative. Dual phases contrast computed tomography (CT) scan and magnetic resonance imaging (MRI) of abdomen are normal which ruled out Insulinoma as well as other possible abdominal malignancy. The CT scan of chest was normal. Base line morning serum cortisol level was $11.8 \mu \mathrm{g} / \mathrm{dL}$ (normal range $5-25 \mu \mathrm{g} / \mathrm{dL}$ ). Serum fasting insulin and $\mathrm{C}$ peptide level were $3.8 \mathrm{IU} / \mathrm{mL}$ (normal range $2-25 \mathrm{IU} / \mathrm{mL})$ and $1.94 \mathrm{ng} / \mathrm{mL}(0.9-7.1 \mathrm{ng} / \mathrm{mL})$. Electroencephalogram showed generalized slow background delta wave activity suggestive of diffuse cerebral dysfunction. Brain MRI (3 Tesla) of T2 and FLAIR sequences showed bilateral symmetrical white matter hyper intensities involving middle cerebellar peduncle (Figure 1A). Diffusion weighted image (DWI) sequences showed hyper intensities in bilateral corona radiata, centrum semiovale and internal capsule (Figure 1B-C). FDG-PET/CT imaging showed decreased FDG uptake in bilateral parietal lobes, basal ganglia, thalamus and cerebellum (Figure 2). He was considered to be hypoglycemic encephalopathy of unknown cause and treated with continuous dextrose solution with frequent high carbohydrate diet. He started improving after two weeks of ICU stay. After four weeks of treatment he started responding and recognizing family members with severe hypo kinetic dysarthria and generalized rigidity. Repeat MRI of brain after four weeks showed resolution of all previous lesions. After two months of admission, he was clinically improved with minimal extra pyramidal symptoms. His both modified rankin scale (MRS) and barthel index scores were five of each at the time of admission which improved to score two and ninety, respectively within a period of two months of hospital stay.
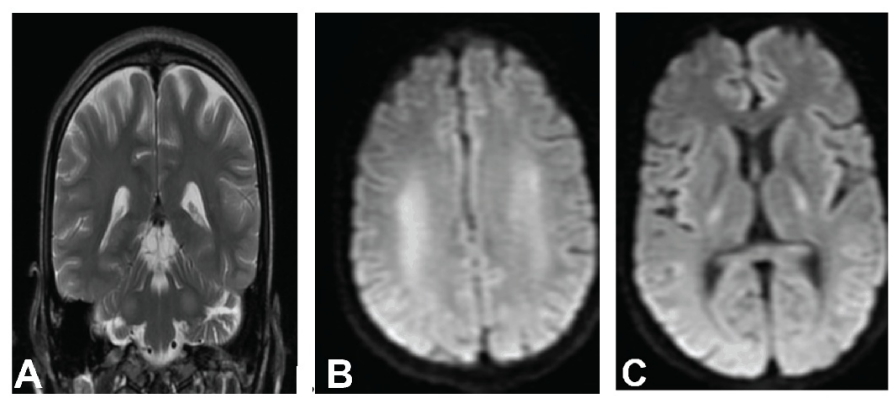

Figure 1: (A) Coronal section of T2-weighted sequence of magnetic resonance imaging of brain showing bilateral symmetrical hyper intensity of middle cerebella peduncle (MCP) (white arrow), (B) Diffusion weighted image showed bilateral symmetrical increased signal intensities in centrum semiovale. (black arrow), (C) Diffusion weighted image showing increased signal intensities in both internal capsules (white triangle).

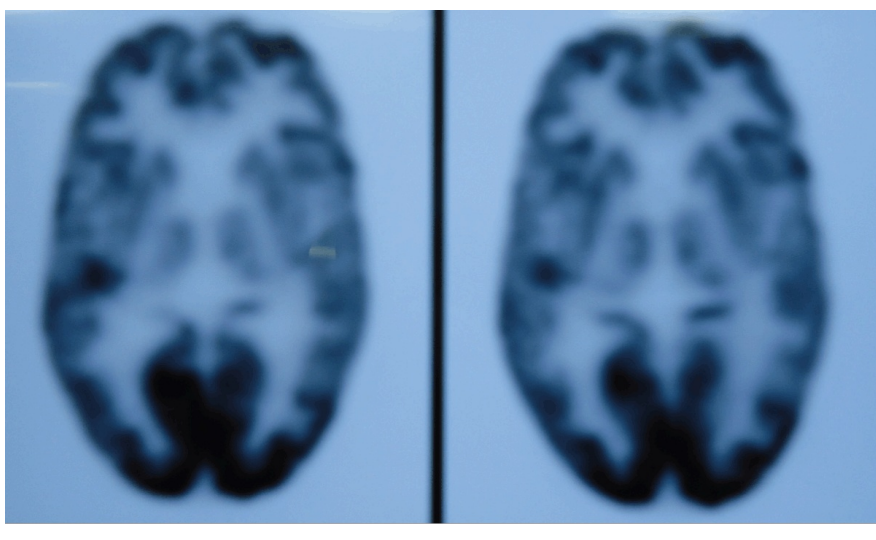

Figure 2: 18F-fluorodeoxyglucose positron emission tomography with computed tomography scan of brain showing decreased fluorodeoxyglucose uptake. 


\section{FOLLOW-UP}

After three months of hospital discharge, he achieved pre morbid state except minimal rigidity of limbs. His speech became clear on conversation. He was maintaining normal blood glucose with high carbohydrate diet.

\section{DISCUSSION}

Though there is no consensus regarding clinical definition of this term, Jens Witsch et al. in their series defined this entity as a clinical state leading to stupor or coma with the blood glucose level of $<50 \mathrm{mg} / \mathrm{dL}$ and persistence of clinical symptoms for $\geq 24 \mathrm{~h}$ despite normalization of blood glucose levels unaccompanied by other systemic complications and possible reasons simulating the same [5]. Most vulnerable area of brain to be affected by hypoglycemia are the subiculum, caudate neurons, hippocampus, the dentate gyrus and superficial cortical layers especially layers 2 and 3 [3].

The exact pathophysiology of hepatic encephalopathy is poorly understood. Hypoglycemic brain produces increase amount of aspartate from oxaloacetate due to lake of acetate in Krebs cycle leading to energy failure of about $25 \%$ of normal leading to selective tissue necrosis and brain damage. Hypoglycemic tissue alkalosis and apoptosis may contribute to the above pathogenesis [6]. Hypoglycemic brain damage usually results due to tissue alkalosis because of increased $\mathrm{pH}$, absence of lactic acid and raised ammonia from deamination of amino acids [9]. It preferentially affects neurons and usually spares axons $[8,9]$. The mechanism of infarction is quite different from that of hypoglycemic brain damage. Decreased brain $\mathrm{pH}$, elevated lactate and reduced cellular redox system are the main culprit in ischemia [7]. Ischemia affects all parts of neurons as well as glial and endothelial tissues $[8,9]$.

Hypoglycemia may have variable clinical presentations. Parkinsonism secondary to hypoglycemic encephalopathy is due to degeneration of dopamine neurons in the substantial nigra pars compacta (SNc), secondary to ischemia or severe hypoglycemia. There is hyper polarization of dopamine neurons in acute brain injury during hypoxia and hypoglycemia. However, the role of excitatory synapses in SNc in this condition is poorly understood [10].

Akinetic-rigid syndrome implies difficulty in initiating movements (bradykinesia or akinesia), rigidity, progressive fatiguing, slow mentation (bradyphrenia) with or without tremor at rest and postural instability [11]. It is caused by diverse etiology. It is important to differentiate idiopathic parkinsonism to secondary parkinsonism because of different therapeutic options. The diagnosis is based entirely on clinical profiles.

In this case, the patient suffered from intermittent hypoglycemia for three years. Later on due to sustained unaware hypoglycemic state, he developed prolonged delirium followed by severe extra pyramidal symptoms in the form of akinesia, rigidity (axial and appendicular), bradyphrenia, and severe hypophonia with postural instability. With replacement of glucose, patient showed clinical improvement and maintained his independent daily activities after three months. We found the following three atypical manifestations in our case:

(1) Akinetic-rigid syndrome secondary to persistent hypoglycemia

(2) Rare neuroimaging findings and

(3) Discordant FDG-PET scans with relation to MRI findings and subsequent recovery of the lesions.

The prognosis of hypoglycemic encephalopathy depends on co morbidities, associated medical complications, early recognition and treatment, extent of neuroimaging findings and its reversibility. The mortality ranges from $23-46 \%$ in various series and when the outcome survived is excellent [5].

\section{CONCLUSION}

Hypoglycemic encephalopathy is a rare emergency which may develop reversible extra pyramidal symptoms like akinetic-rigid syndrome. Neuro imaging correlation is not always possible to describe the temporal profile of the patients. Besides routine evaluation for hypoglycemia, neuroimaging such as magnetic resonance imaging and 18Ffluorodeoxyglucose positron emission tomography fused with computed tomography scan may contribute to recognize the disease process and its outcome

$* * * * * * * * *$

\section{Author Contributions}

Akhila Kumar Panda - Conception and design, Acquisition of data, Analysis and interpretation of data, Critical revision of the article, Final approval of the version to be published

Suman Kushwaha - Analysis and interpretation of data, drafting the article, Final approval of the version to be published

Aldrin Anthony Dung Dung - Acquisition of data, drafting the article, Final approval of the version to be published

\section{Guarantor}

The corresponding author is the guarantor of submission.

\section{Conflict of Interest}

Authors declare no conflict of interest.

\section{Copyright}

(C) Akhila Kumar Panda et al. 2013; This article is distributed under the terms of Creative Commons attribution 3.0 License which permits unrestricted use, distribution and reproduction in any means provided the original authors and original publisher are properly 
credited. (Please see www.ijcasereportsandimages.com /copyright-policy.php for more information.)

\section{Acknowledgements}

We thank to Vachan Jayant Mehta, and Seema Malik for their help in preparing the manuscript.

\section{Abbreviations}

SNc : Substantial nigra pars compacta

CNS : Central nervous system

MRI : Magnetic resonance imaging

MCP : Middle cerebellar peduncl

FDG PET CT : 18F- fluorodeoxyglucose positron emission tomography fused with computed tomography

CT : Computed tomogram

CSF : Cerebrospinal fluid

DNA : Deoxy ribonucleic acid

FLAIR : Fluid attenuated inversion recovery

HIV : Human immune deficiency virus

\section{REFERENCES}

1. Laing SP, Swerdlow AJ, Slater SD, et al. The British Diabetic Association Cohort Study. II. Cause-specific mortality in patients with insulin-treated diabetes mellitus. Diabet Med 1999 Jun;16(6):466-71.

2. Cryer PE, Davis SN, Shamoon H. Hypoglycemia in diabetes. Diabetes Care 2003 Jun;26(6):1902-12.
3. Auer RN, Wieloch T, Olsson Y, Siesjo BK. The distribution of hypoglycemic brain damage. Acta Neuropathol 1984;64(3):177-91.

4. Guettier JM, Gorden P. Hypoglycemia. Endocrinol Metab Clin North Am 2006 Dec;35(4):753-66.

5. Witsch J, Neugebauer H, Flechsenhar J, Jüttler E. Hypoglycemic encephalopathy: a case series and literature review on outcome determination. J Neurol 2012 Oct;259(10):2172-81.

6. Auer RN. Hypoglycemic Brain Damage. Metab Brain Dis 2004 Dec;19(3-4):169-75.

7. Kang EG, Jeon SJ, Choi SS, Song CJ, Yu IK Diffusion MR imaging of hypoglycemic encephalopathy. Am J Neuroradiol 2010;31(3):559-64.

8. Helgason CM. Blood glucose and stroke. Stroke 1988 Aug;19(8):1049-53.

9. Auer RN. Hypoglycemic brain damage. Forensic Sci Int 2004;146(2-3):105-10.

10. Singh V, Carman M, Roeper J, Bonci A. Brief ischemia causes long-term depression in midbrain dopamine neurons. Eur J Neurosci 2007;26(6):1489-99.

11. Quinn N. Parkinsonism--recognition and differential diagnosis. BMJ 1995;310(6977):447-52.
Access full text article on other devices

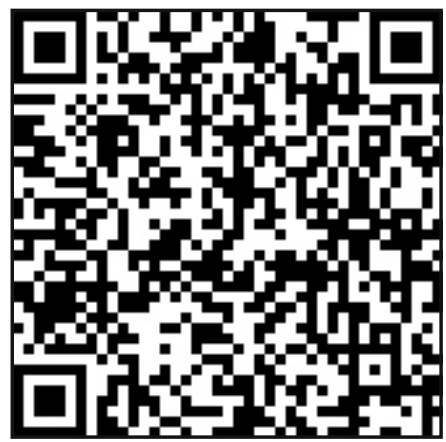

Access PDF of article on other devices

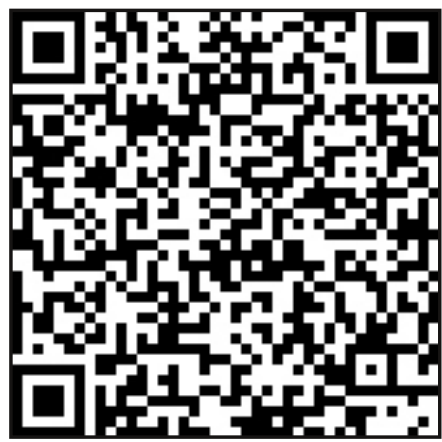

